\title{
Human Leukocyte Antigen Class I and II Alleles and Overall Survival in Diffuse Large B-Cell Lymphoma and Follicular Lymphoma
}

Yani Lu, ${ }^{1}$ Amr M. Abdou, ${ }^{2}$ James R. Cerhan, ${ }^{3}$ Lindsay M. Morton, ${ }^{4}$ Richard K. Severson, ${ }^{5}$ Scott Davis, ${ }^{6}$ Wendy Cozen, ${ }^{7}$ Nathaniel Rothman, ${ }^{4}$ Leslie Bernstein, ${ }^{1}$ Stephen Chanock, ${ }^{4}$ Patricia Hartge, ${ }^{4}$ and Sophia S. Wang ${ }^{1}$

${ }^{1}$ Division of Cancer Etiology, Department of Population Sciences, Beckman Research Institute, City of Hope, Duarte, CA 91010, USA

${ }^{2}$ Department of Microbiology and Immunology, National Research Center, Cairo, Egypt

${ }^{3}$ Mayo Clinic, College of Medicine, Rochester, MN 55905, USA

${ }^{4}$ Division of Cancer Epidemiology and Genetics, National Cancer Institute, NIH, Rockville, MD 20852, USA

${ }^{5}$ Department of Family Medicine and Public Health Sciences, Karmanos Cancer Institute, Wayne State University, Detroit, MI 48202, USA

${ }^{6}$ Fred Hutchinson Cancer Research Center and University of Washington, Seattle, WA 98109-4433, USA

${ }^{7}$ Norris Comprehensive Cancer Center, University of Southern California, Los Angeles, CA 90033, USA

Received 29 July 2011; Accepted 3 October 2011

Academic Editor: Edward Benz

Genetic variation in the 6p21 chromosomal region, including human leukocyte antigen $(H L A)$ genes and tumor necrosis factor $(T N F)$, has been linked to both etiology and clinical outcomes of lymphomas. We estimated the effects of $H L A$ class I (A, B, and $\mathrm{C})$, class II DRB1 alleles, and the ancestral haplotype $(\mathrm{AH}) 8.1$ (HLA $\left.A^{*} 01-B^{*} 08-D R B 1^{*} 03-T N F-308 A\right)$ on overall survival (OS) among patients with diffuse large B-cell lymphoma (DLBCL) and follicular lymphoma (FL) in a population-based study of non-Hodgkin lymphoma. During a median followup of 89 months, $31 \%$ (52 of 166) DLBCL and 28\% (46 of 165) FL patients died. Using multivariate Cox regression models, we observed statistically significant associations between genetic variants and survival: $H L A-C w^{*}$ 07:01 was associated with poorer OS among DLBCL patients (Hazard ratio [HR] = 1.76, 95\% confidence interval $[\mathrm{Cl}]=1.01-3.05) ; H L A-A^{*} 01: 01$ was associated with poorer OS (HR $=2.23,95 \% \mathrm{Cl}=1.24-4.01)$, and $H L A-D R B 1 * 13(\mathrm{HR}=0.12,95 \% \mathrm{Cl}=0.02-0.90)$ and $H L A-B$ Bw4 $(\mathrm{HR}=0.36,95 \% \mathrm{Cl}=0.20-0.63)$ with better OS among $\mathrm{FL}$ patients. These results support a role for $H L A$ in the prognosis of DLBCL and FL and represent a promising class of prognostic factors that warrants further evaluation.

KEYWORDS: human leukocyte antigen, tumor necrosis factor, diffuse large B-cell lymphoma, follicular lymphoma, survival 


\section{INTRODUCTION}

Diffuse large B-cell lymphoma (DLBCL) and follicular lymphoma (FL) are the two most commonly diagnosed subtypes of non-Hodgkin lymphoma (NHL) in the United States. The established prognostic factors for DLBCL as delineated in the International Prognostic Factor Index (IPI) include age, clinical stage, number of extranodal sites, performance score, and lactate dehydrogenase level [1]; the established prognostic factors for FL as validated by the Follicular Lymphoma International Prognostic Index (FLIPI) include age, clinical stage, number of nodal areas, hemoglobin level, and lactate dehydrogenase level [2]. However, the clinical course of both diseases is variable, and both IPI and FLIPI predict prognosis incompletely. Patient genetic variation may improve predicted prognosis after diagnosis with DLBCL and FL.

Genetic variation in the 6p21 chromosomal region has been linked to both etiology and clinical courses of lymphomas and related risk factors. The $6 \mathrm{p} 21$ chromosomal region houses the major histocompatibility complex, which includes the human leukocyte antigen (HLA) genes and tumor necrosis factor (TNF). TNF and the ancestral HLA haplotype 8.1, which includes TNF, (AH 8.1: HLA $A^{*} 01-B^{*} 08$ $D R B 1^{*} 03-T N F-308 A$ ) have been implicated in DLBCL etiology in epidemiology studies [3] and NHL survival in a series of clinical studies [4-7]. In our etiological investigations, we have also reported associations between $H L A-D R B 1^{*} 04: 01$ with DLBCL risk and $H L A-D R B 1^{*} 13$ and $H L A-D R B 1^{*} 01: 01$ with FL risk [8], the latter of which has been corroborated in a genome-wide association study (GWAS) [9]. In addition, all $H L A-B$ alleles can be further grouped (mutually exclusively) as $H L A-B B w 4$ or $B w 6$ based on amino acid residues at positions 79-83, and HLA-B Bw4 has been associated with and has delayed progression to acquired immunodeficiency syndrome (AIDS) among those with the human immunodeficiency virus (HIV), a known risk factor for NHL [10].

Here, we evaluate whether these previously implicated alleles, other $H L A$ class I (A, B, C) and class II $D R B 1$ alleles, and AH 8.1 are associated with DLBCL and FL survival in a multicenter population-based study of NHL in the United States.

\section{METHODS}

Details of the National Cancer Institute-Surveillance, Epidemiology and End Results (NCI-SEER) NHL population-based case-control study have been described elsewhere [4, 8, 11]. Briefly, 1,321 newly diagnosed and histologically confirmed NHL patients, aged 20-74 years, were identified in four SEER registries (Iowa; Detroit, Mich; Los Angeles, Calif; Seattle, Wash) during July 1998-June 2000. Patients had no evidence of HIV infection but testing for HIV was not conducted as part of the study. All participants provided written informed consent and completed an in-person interview; $1172(89 \%)$ provided either a venous blood sample $(n=773)$ or mouthwash buccal cell sample $(n=399)$. Of 365 DLBCL ( 215 with blood) and 278 FL (192 with blood) cases enrolled in the study, the present analysis comprises the 179 DLBCL and 180 FL cases who had complete clinical and demographic information and sufficient DNA for HLA allelotyping. Characteristics for those with sufficient DNA and those without sufficient DNA were similar (data not shown), making any potential biases for our results based on patients with sufficient DNA unlikely.

Demographic information, including age, sex, race, Hispanic ethnicity, and education level, was derived from patients' interviews as part of the case-control study. Clinical information, including date of diagnosis, histology, stage, presence of B-symptoms, first course of therapy, date of last followup, and vital status, was derived from linkages to the SEER registry databases at each study site in 2008. Data on first course of therapy included use of single or multiagent chemotherapy, radiation, other therapies exclusive of chemotherapy and/or radiation, and no therapy (observation); information on individual agents and doses was not available. The SEER registries collect date and cause of death but do not collect data on treatment response or disease recurrence or progression.

Pathology information was derived from abstracted reports by the local diagnosing pathologist. All cases were coded according to the International Classification of Diseases-Oncology (ICD-O), 2nd Edition, 
and updated to the WHO/ICD-O-3. DLBCL was defined as ICD-O 9678, 9679, 9680, and 9684 and FL as 9690-9691, 9695, and 9698.

DNA was extracted using Puregene Autopure DNA extraction kits (Gentra Systems, Minneapolis, Minn). Four-digit HLA Class I (A, B, and C) and Class II genotyping (DRB1) was conducted at NCIFrederick (Frederick, MD) according to sequence-specific oligonucleotide probe (SSOP) hybridization and sequence-based typing protocols developed by the 13th International Histocompatibility Workshop. Agreement for quality control duplicates $(n=100)$ was $\geq 99 \%$. $H L A$ alleles were defined as presence (if the individual had either one or two copies of the allele) or absence of the specific allele (defined as having no copies of the allele). TNF genotyping was conducted at the National Cancer Institute Core Genotying facility (Gaithersburg, MD, USA) using the Taqman (Foster City, Calif, USA) PLATFORM. TNF G-308A was defined by genotype (GG (referent), GA, and AA). Haplotypes for $H L A-A-B-D R$ were determined using "FastHap", which determines haplotypes by expectation maximization (http://home.ncifcrf.gov/ccr/lgd/ bioinformatics/index_n.asp).

We fit Cox proportional hazard regression models to estimate hazard ratios (HRs) and 95\% confidence intervals (95\% CIs) for the association of each individual HLA loci (e.g., HLA-A, -B, $-C$, or $-D R B 1)$ and haplotype with overall survival, adjusting for demographic and clinical factors. Demographic and clinical factors were modeled as one risk score, which was a linear combination of age $(<60$ versus $\geq 60$ years), sex, race (white and all other), study center (Detroit, Iowa, Los Angeles, and Seattle), and years of education $(<12,12-15$, and $\geq 16$ years), tumor stage (local, regional, distant, and missing), presence of $\mathrm{B}$ symptoms (no, yes, missing), and type of initial therapy (chemotherapy plus radiation, chemotherapy plus other therapy, radiation only, all other, or missing therapy) $[4,11]$. Analyses were also conducted restricted to non-Hispanic Caucasians. Two-sided $P$ values were reported. All analyses were conducted using SAS 9.1.3 (SAS Institute, Cary, NC, USA).

\section{RESULTS}

Descriptive characteristics (demographic and clinical features) of our population are shown in Table 1. The median age at diagnosis was 58 years for DLBCL patients (range, 20-74) and 56 years for FL patients (range, 28-74) [8]. Male patients comprised 55\% and 49\% of DLBCL and FL, respectively. Ninety-two percent DLBCL and FL patients identified themselves as non-Hispanic Caucasians. Thirty-eight percent of DLBCL and $56 \%$ of FL patients had advanced stage (distant) disease. Twenty-four percent of DLBCL and $14 \%$ of FL patients had B symptoms. Chemotherapy-based regimen was the most common initial therapy. With a median followup of 89 months (range, 7-112 months), 58 of 179 (32\%) DLBCL and 48 of 180 (27\%) FL patients died.

\section{HLA Class I and II Alleles}

Among FL cases, presence of $H L A-A^{*} 01: 01$ was associated with poorer $\mathrm{OS}(\mathrm{HR}=2.23,95 \% \mathrm{CI}=1.24$ 4.01), whereas the presence of $H L A-B B w 4(\mathrm{HR}=0.36,95 \% \mathrm{CI}=0.20-0.63)$ and $H L A-D R B I^{*} 13(\mathrm{HR}=$ $0.12,95 \% \mathrm{CI}=0.02-0.90$ ) was associated with better OS (Table 2). The presence of $H L A-C w^{*} 07: 01$ was associated with poorer OS among DLBCL patients ( $\mathrm{HR}=1.76,95 \% \mathrm{CI}=1.01-3.05)$.

\section{AH 8.1}

The AH 8.1 (HLA-A*01- $\left.B^{*} 08-D R^{*} 03-T N F-308 A\right)$ was associated with poorer OS among patients with FL, likely due to a strong association with the $H L A-A^{*} 01-B^{*} 08-D R^{*} 03$ haplotype $(\mathrm{HR}=2.19,95 \% \mathrm{CI}=1.08$ 4.45), whereas TNF G-308A was not associated with survival after FL (Table 2). In contrast, among patients with DLBCL, the AH 8.1 and HLA-A were not associated with OS, but the presence of the TNF-308A allele was associated with poorer OS (HR for GA/AA versus $\mathrm{GG}=1.79,95 \% \mathrm{CI}=1.02-3.14$ ).

Corresponding survival curves for each HLA allele and AH 8.1 association for DLBCL and FL are shown in the Figure 1. 
TABLE 1: Demographic and clinical characteristics of DLBCL and FL patients from the NCI-SEER NHL study for which HLA typing was conducted.

\begin{tabular}{lcc}
\hline Characteristics & DLBCL & FL \\
& $n(\%)$ & $n(\%)$ \\
\hline Sex & & \\
$\quad$ Male & $99(55.3)$ & $89(49.4)$ \\
Female & $80(44.7)$ & $91(50.6)$ \\
Age (years) & & $103(57.2)$ \\
$\quad<60$ & $93(52.0)$ & $77(42.8)$ \\
$60+$ & $86(48.0)$ & \\
Race & & $165(91.7)$ \\
Non-Hispanic Caucasian & $165(92.2)$ & $15(8.3)$ \\
Other & $14(7.8)$ & $16(8.9)$ \\
Study Center & & $76(42.2)$ \\
Detroit & $19(10.6)$ & $30(16.7)$ \\
Iowa & $67(37.4)$ & $58(32.2)$ \\
Los Angeles & $29(16.2)$ & $11(6.1)$ \\
Seattle & $64(35.8)$ & $118(65.6)$ \\
Education (years) & & $51(28.3)$ \\
$\quad<12$ & $16(8.9)$ & $154(85.6)$ \\
12-15 & $118(65.9)$ & $26(14.4)$ \\
16+ & $45(25.1)$ & $44(24.4)$ \\
Stage & & $25(13.9)$ \\
Local & $69(38.6)$ & $101(56.1)$ \\
Regional & $39(21.8)$ & $10(5.6)$ \\
Distant & $38(38.0)$ & \\
Unknown & $3(1.7)$ & \\
Presence of B symptoms & $136(76.0)$ & \\
No & $43(24.0)$ & \\
$\quad$ Yes & & \\
\hline
\end{tabular}

\section{DISCUSSION}

Our data suggest that specific $H L A$ alleles are associated with survival among DLBCL and FL patients. Specifically, $H L A-C w^{*}$ 07:01 was associated with poorer OS among DLBCL patients, and HLA-A*01:01 was associated with poorer OS and $H L A-D R B I^{*} 13$ and $H L A-B B w 4$ with better OS among FL patients. Overall, the results add to the growing body of evidence of a role for $H L A$ in the prognosis of these subtypes.

Our results agree with various clinical studies [5-7, 12] in which TNF $G-308 A$ and AH 8.1 have been reported to influence clinical course and outcome of NHL overall. In one analysis restricted to DLBCL patients, TNF- $\alpha$ in plasma predicted DLBCL survival [12], a pattern consistent with our study results that TNF G-308A increases the risk of death among DLBCL patients. In that study, the authors also reported that AH 8.1 predicted overall survival of NHL patients, and that $H L A-A^{*} 01$ and $H L A-B^{*} 08$ were associated with prognosis [7]. In this study, AH 8.1 was associated with poorer OS among FL patients, a result that our data suggest to be largely driven by the association with $H L A-A^{*} 01: 01$. Taken together, our results support the hypothesis that chronic inflammation contributes to poorer prognosis of DLBCL, but other mechanisms of immune dysregulation may further be considered for FL prognosis. 
TABLE 2: HLA class I and II alleles and haplotypes and their associations with overall survival among diffuse large B-cell lymphoma (DLBCL) and follicular lymphoma (FL) patients.

\begin{tabular}{|c|c|c|c|c|c|c|c|}
\hline \multirow[b]{2}{*}{ Allele/Haplotype } & \multirow[b]{2}{*}{ Status } & \multicolumn{3}{|c|}{ DLBCL } & \multicolumn{3}{|c|}{ FL } \\
\hline & & $\begin{array}{c}\text { Total } \\
\text { no. }\end{array}$ & $\begin{array}{l}\text { No. of } \\
\text { deaths }\end{array}$ & $\mathrm{HR}(95 \% \mathrm{CI})$ & $\begin{array}{c}\text { Total } \\
\text { no. }\end{array}$ & $\begin{array}{l}\text { No. of } \\
\text { deaths }\end{array}$ & $\mathrm{HR}(95 \% \mathrm{CI})$ \\
\hline \multicolumn{8}{|l|}{ HLA class I } \\
\hline \multirow{2}{*}{$H L A-A^{*} 01: 01$} & Absent & 107 & 31 & 1.00 (ref) & 109 & 23 & 1.00 (ref) \\
\hline & Present & 59 & 21 & $1.30(0.74-2.26)$ & 54 & 22 & $2.23(1.24-4.01)$ \\
\hline \multirow{2}{*}{$H L A-B^{*} 08: 01$} & Absent & 123 & 35 & 1.00 (ref) & 123 & 32 & 1.0 (ref) \\
\hline & Present & 42 & 17 & $1.40(0.78-2.51)$ & 42 & 14 & $1.39(0.74-2.61)$ \\
\hline \multirow{2}{*}{$H L A-C w^{*} 07: 01$} & Absent & 111 & 28 & 1.00 (ref) & 116 & 32 & 1.00 (ref) \\
\hline & Present & 55 & 24 & $1.76(1.01-3.05)$ & 49 & 14 & $1.12(0.60-2.10)$ \\
\hline \multirow{2}{*}{$H L A-B B w 4$} & Absent & 73 & 27 & 1.00 (ref) & 55 & 25 & 1.00 (ref) \\
\hline & Present & 106 & 31 & $0.97(0.58-1.64)$ & 125 & 23 & $0.36(0.20-0.63)$ \\
\hline \multicolumn{8}{|l|}{ HLA class II } \\
\hline \multirow{2}{*}{$H L A-D R B 1^{*} 01: 01$} & Absent & 134 & 43 & 1.00 (ref) & 118 & 32 & 1.00 (ref) \\
\hline & Present & 32 & 9 & $0.94(0.46-1.94)$ & 47 & 14 & $1.12(0.59-2.10)$ \\
\hline \multirow{2}{*}{$H L A-D R B 1^{*} 03: 01$} & Absent & 121 & 34 & 1.00 (ref) & 127 & 33 & 1.00 (ref) \\
\hline & Present & 45 & 18 & $1.41(0.79-2.50)$ & 38 & 13 & $1.33(0.70-2.54)$ \\
\hline \multirow{2}{*}{$H L A-D R B 1^{*} 13$} & Absent & 137 & 39 & 1.00 (ref) & 146 & 47 & 1.00 (ref) \\
\hline & Present & 29 & 13 & $1.34(0.71-2.55)$ & 19 & 1 & $0.12(0.02-0.90)$ \\
\hline \multicolumn{8}{|l|}{ AH 8.1} \\
\hline \multirow{2}{*}{$H L A-A^{*} 01-B^{*} 08-D R^{*} 03$} & Absent & 135 & 40 & 1.00 (ref) & 143 & 35 & 1.00 (ref) \\
\hline & Present & 30 & 12 & $1.46(0.76-2.80)$ & 20 & 10 & $2.19(1.08-4.45)$ \\
\hline \multirow{4}{*}{$T N F$ G-308A } & GG & 98 & 26 & 1.00 (ref) & 107 & 28 & 1.00 (ref) \\
\hline & GA & 53 & 21 & $1.66(0.93-2.97)$ & 42 & 15 & $1.52(0.81-2.86)$ \\
\hline & AA & 6 & 3 & $3.81(1.13-12.9)$ & 4 & 1 & $0.84(0.11-6.19)$ \\
\hline & GA/AA & 59 & 24 & $1.79(1.02-3.14)$ & 46 & 16 & $1.45(0.78-2.68)$ \\
\hline$H L A-A^{*} 01-B^{*} 08-$ & Absent & 132 & 39 & 1.00 (ref) & 135 & 34 & 1.00 (ref) \\
\hline$D R^{*} 03-T N F-A$ & Present & 29 & 11 & $1.39(0.71-2.73)$ & 20 & 10 & $2.13(1.05-4.34)$ \\
\hline
\end{tabular}

All models adjusted for the risk score that combined both demographic and clinical information.

The total numbers for each allele may differ due to laboratory interpretation of each allele which was done individually (e.g., ambiguous results for each allele were excluded from analysis rather than presumed to be absent).

We previously reported that the $H L A-D R B 1^{*} 13$ group of alleles were associated with decreased risk of developing FL. The newly identified association with better survival, if confirmed in other data, would implicate an important $H L A$ allele in both FL etiology and survival. We also found better OS among FL patients with $H L A-B B w 4$, suggesting possible similarities in mechanisms by which $H L A-B B w 4$ slows the progression to AIDs. On the other hand, we did not observe a joint relationship between $H L A-B B w 4$ with the KIR receptor (KIR3DS1), as has been reported for AIDS outcomes (data not shown) [10]. These results support the potential role for the innate immune response and immune surveillance as important aspects for FL etiology and prognosis.

We also report here that $H L A-C w^{*} 07: 01$ was associated with poorer OS among DLBCL patients, which was not found in a previous study [7]. This inconsistency could reflect chance, heterogeneity of 
DLBCL

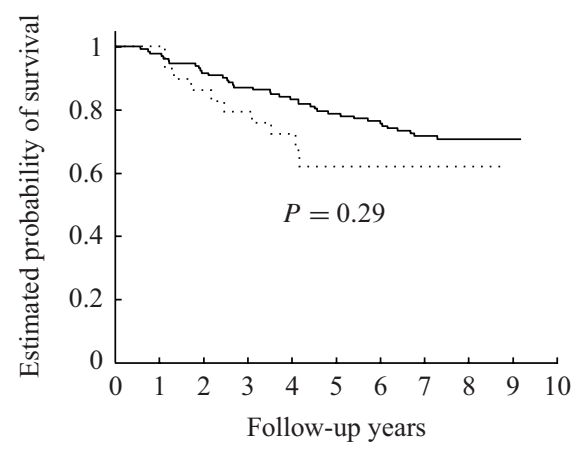

FL

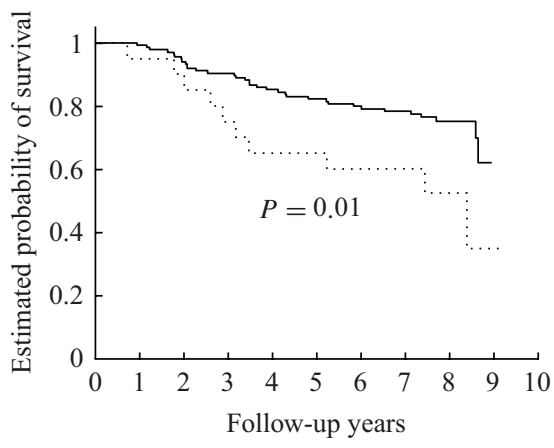

(a) $A H 8.1$
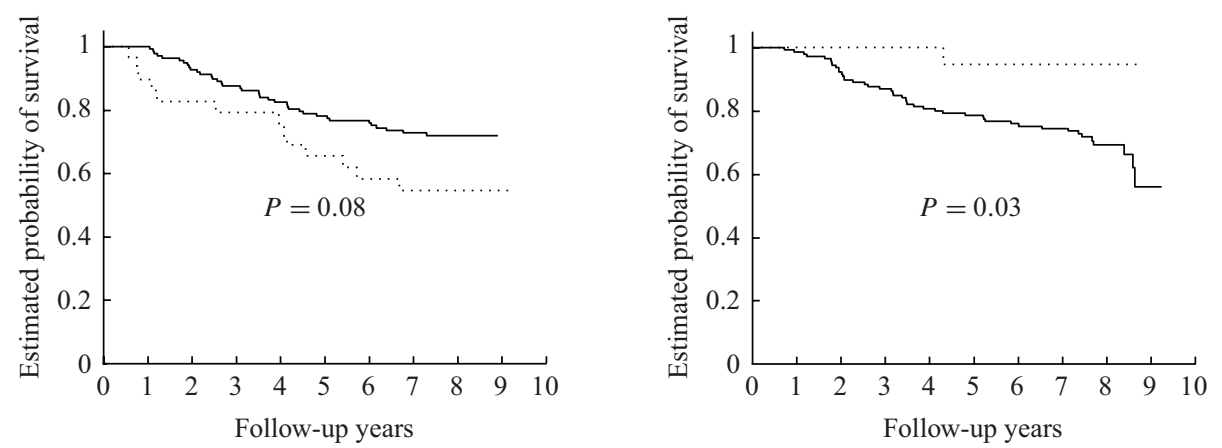

(b) $H L A-D R B 1^{*} 13$
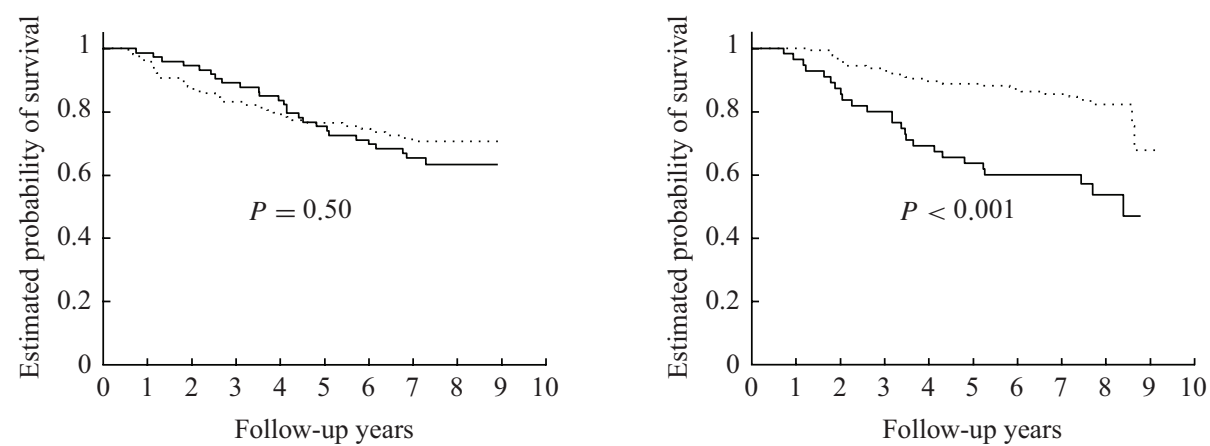

(c) $H L A-B w 4$
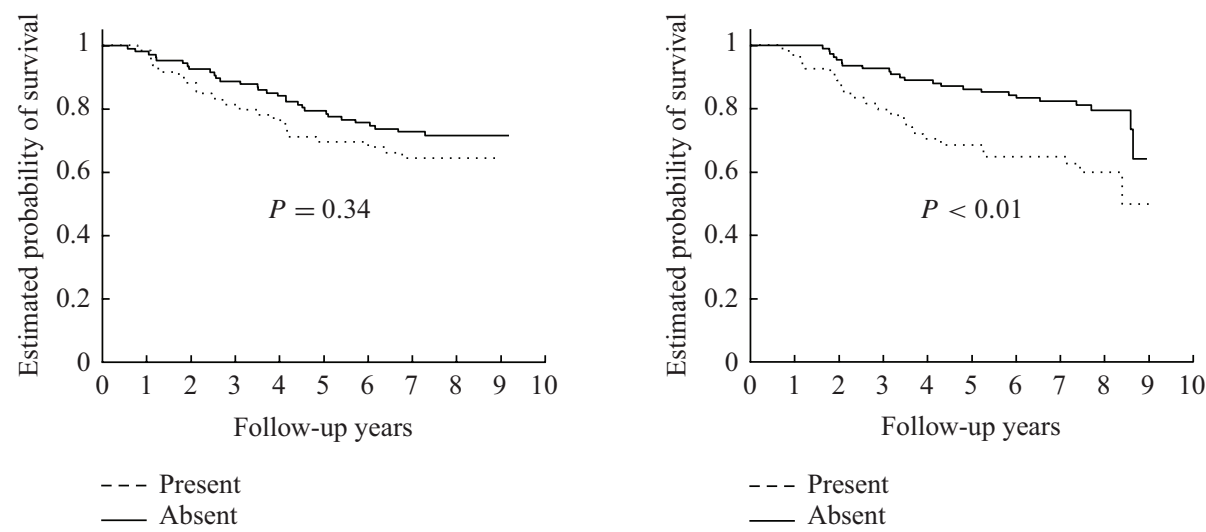

(d) HLA-A*01:01

FIGURE 1: Continued. 

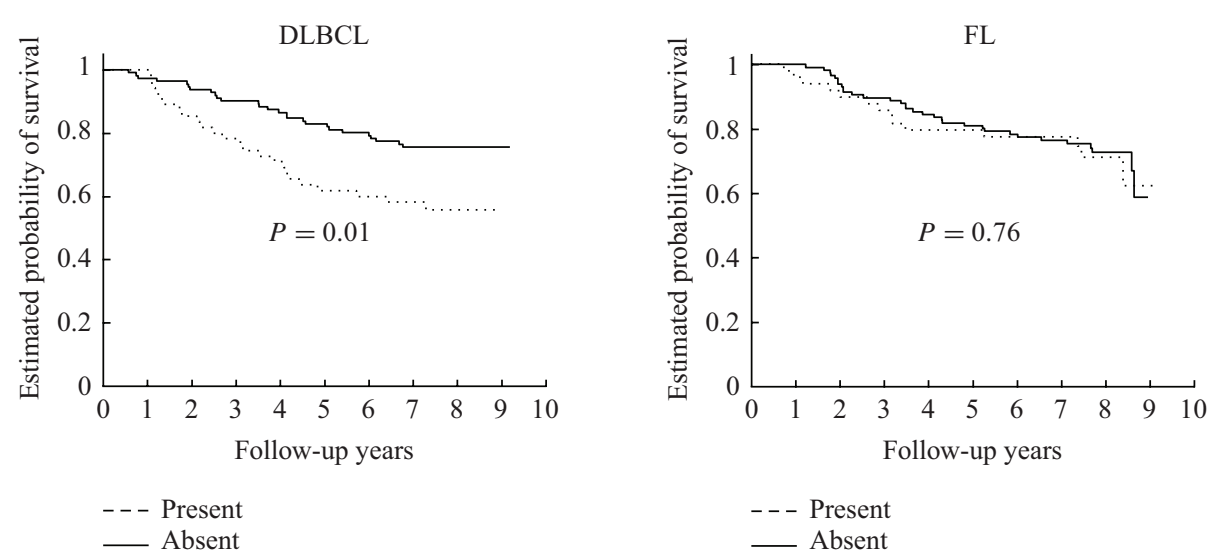

(e) HLA-Cw07:01

FIGURE 1: Survival curves and Kaplan-Meier $P$-values among patients with DLBCL and FL by HLA alleles and haplotype: (a) AH 8.1; (b) HLA-DRB1*13; (c) HLA-Bw4; (d) HLA-A*01:01; (e) HLA-Cw07:01.

the NHL definition in the previous clinical studies where the authors examined the risk of NHL overall instead of by subtype [5-7], or dilution of the moderate association in the broad NHL definition compared to analysis specific to DLBCL.

We note that most of of the HLA alleles identified as potentially relevant for etiology were not predictive for survival, suggesting distinct functions for these genetic variations [8]. Alleles previously found associated with NHL etiology in our study included $H L A-A^{*} 26: 01, A^{*} 29: 02, B^{*}$ 51:01, $C w^{*} 03: 04$, $C w^{*}$ 15:02, $D R B 1^{*} 04: 01$, and $H L A-D R B 1^{*} 01: 01$ [8], none of which were identified as associated with survival after DLBCL or FL.

An important strength of our study was the population-based ascertainment of newly diagnosed cases, making our results more applicable to community-based treatment programs. Furthermore, we were able to evaluate the results by subtype, whereas most clinical studies have only assessed the association for NHL overall [5-7]. One important potential limitation apart from sample size is the lack of detailed data on prognostic factors or treatment and that we missed most DLBCL deaths that occurred in the first year. By using age, stage, B symptoms, and treatment class, we, nonetheless, could predict survival with a level of ability similar to the IPI for a large study of DLBCL and FL patients $[4,11]$. Because all patients were initially treated before the widespread use of rituximab, it will be important to evaluate a patient population treated with rituximab alone (for both DLBCL and FL) and in combination with CHOP chemotherapy (for DLBCL).

In summary, our results support a role for $H L A$ in overall survival of DLBCL and FL patients, after accounting for demographic and clinical factors. Our results are consistent with the emerging significance of the complex host genetic background in progression of each NHL subtype. Host immunogenetics, particularly the major histocompatibility region that houses a number of immune genes including $H L A$, represent a promising class of prognostic factors that warrant further evaluation.

\section{AUTHORS' CONTRIBUTIONS}

Y. Lu and S. S. Wang developed the research question. J. R. Cerhan, W. Cozen, N. Rothman, S. Chanock, R. K. Severson, L. Bernstein, P. Hartge designed and conducted the parent NHL case-control study upon which this analysis was based. A. M. Abdou performed the HLA genotyping. Y. Lu and S. S. Wang conducted data analysis. All authors participated in manuscript preparation. 


\section{ACKNOWLEDGMENTS}

The authors thank Peter Hui of the Information Management Services, Inc. for programming support. They also gratefully acknowledge the contributions of the staff and scientists at the SEER centers of Iowa, Los Angeles, Detroit, and Seattle for the conduct of the study's field effort. The NCI-SEER study was supported by the Intramural Research Program of the NIH (NCI) and by Public Health Service (PHS) Contracts nos. N01-PC-65064, N01-PC-67008, N01-PC-67009, N01-PC-67010, and N02-PC-71105; the survival study was funded by R01 CA96704. HLA typing for the study was funded with federal funds from the National Cancer Institute, National Institutes of Health, under Contract no. HHSN261200800001E and in part by the Intramural Research Program of the NIH, National Cancer Institute. The content of this paper does not necessarily reflect the views or policies of the Department of Health and Human Services, nor does mention of trade names, commercial products, or organizations imply endorsement by the US Government.

\section{REFERENCES}

[1] R. D. Gascoyne, "Emerging prognostic factors in diffuse large B cell lymphoma," Current Opinion in Oncology, vol. 16, no. 5, pp. 436-441, 2004.

[2] P. Solal-Céligny, P. Roy, P. Colombat et al., "Follicular lymphoma international prognostic index," Blood, vol. 104, no. 5, pp. 1258-1265, 2004.

[3] A. M. Abdou, X. Gao, W. Cozen et al., "Human leukocyte antigen (HLA) A1-B8-DR3 (8.1) haplotype, tumor necrosis factor (TNF) G-308A, and risk of non-Hodgkin lymphoma," Leukemia, vol. 24, no. 5, pp. 1055-1058, 2010.

[4] T. M. Habermann, S. S. Wang, M. J. Maurer et al., "Host immune gene polymorphisms in combination with clinical and demographic factors predict late survival in diffuse large B-cell lymphoma patients in the pre-rituximab era," Blood, vol. 112, no. 7, pp. 2694-2702, 2008.

[5] P. Juszczyński, E. Kalinka, G. Woszczek et al., "The role of genetic polymorphisms within tumor necrosis factor promoter gene in non-Hodgkin's lymphomas," Przeglad Lekarski, vol. 62, no. 12, pp. 1417-1421, 2005.

[6] E. Kalinka, P. Juszczyński, G. Woszczek et al., "The role of HLA DRB1 genetic polymorphisms in non-Hodgkin's lymphomas," Przeglad Lekarski, vol. 62, no. 12, pp. 1422-1426, 2005.

[7] J. Nowak, E. Kalinka-Warzocha, P. Juszczynski et al., "Association of human leukocyte antigen ancestral haplotype 8.1 with adverse outcome of non-Hodgkin's lymphoma," Genes Chromosomes and Cancer, vol. 46, no. 5, pp. 500-507, 2007.

[8] S. S. Wang, A. M. Abdou, L. M. Morton et al., "Human leukocyte antigen class I and II alleles in non-Hodgkin lymphoma etiology," Blood, vol. 115, no. 23, pp. 4820-4823, 2010.

[9] L. Conde, E. Halperin, N. K. Akers et al., "Genome-wide association study of follicular lymphoma identifies a risk locus at 6p21.32," Nature Genetics, vol. 42, no. 8, pp. 661-664, 2010.

[10] M. P. Martin, X. Gao, J. H. Lee et al., "Epistatic interaction between KIR3DS1 and HLA-B delays the progression to AIDS," Nature Genetics, vol. 31, no. 4, pp. 429-434, 2002.

[11] J. R. Cerhan, S. Wang, M. J. Maurer et al., "Prognostic significance of host immune gene polymorphisms in follicular lymphoma survival," Blood, vol. 109, no. 12, pp. 5439-5446, 2007.

[12] E. Lech-Maranda, J. Bienvenu, F. Broussais-Guillaumot et al., "Plasma TNF- $\alpha$ and IL-10 level-based prognostic model predicts outcome of patients with diffuse large B-cell lymphoma in different risk groups defined by the international prognostic index," Archivum Immunologiae et Therapiae Experimentalis, vol. 58, no. 2, pp. 131$141,2010$.

\section{This article should be cited as follows:}

Yani Lu, Amr M. Abdou, James R. Cerhan, Lindsay M. Morton, Richard K. Severson, Scott Davis, Wendy Cozen, Nathaniel Rothman, Leslie Bernstein, Stephen Chanock, Patricia Hartge, and Sophia S. Wang, 
"Human Leukocyte Antigen Class I and II Alleles and Overall Survival in Diffuse Large B-Cell Lymphoma and Follicular Lymphoma," TheScientificWorldJOURNAL, vol. 11, pp. 2062-2070, 2011. 


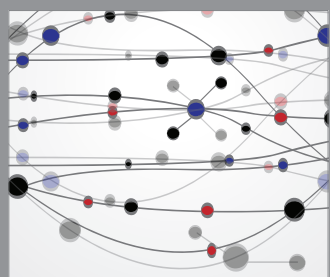

The Scientific World Journal
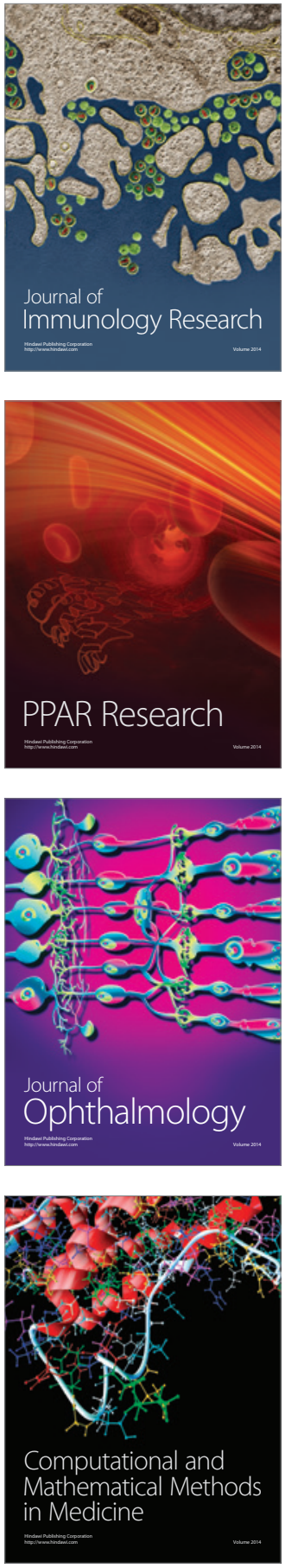

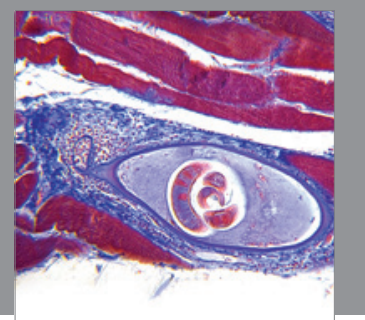

Gastroenterology

Research and Practice
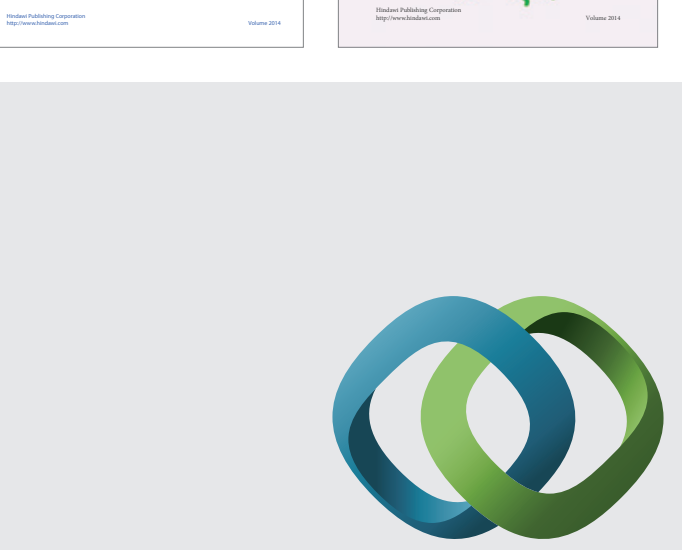

\section{Hindawi}

Submit your manuscripts at

http://www.hindawi.com
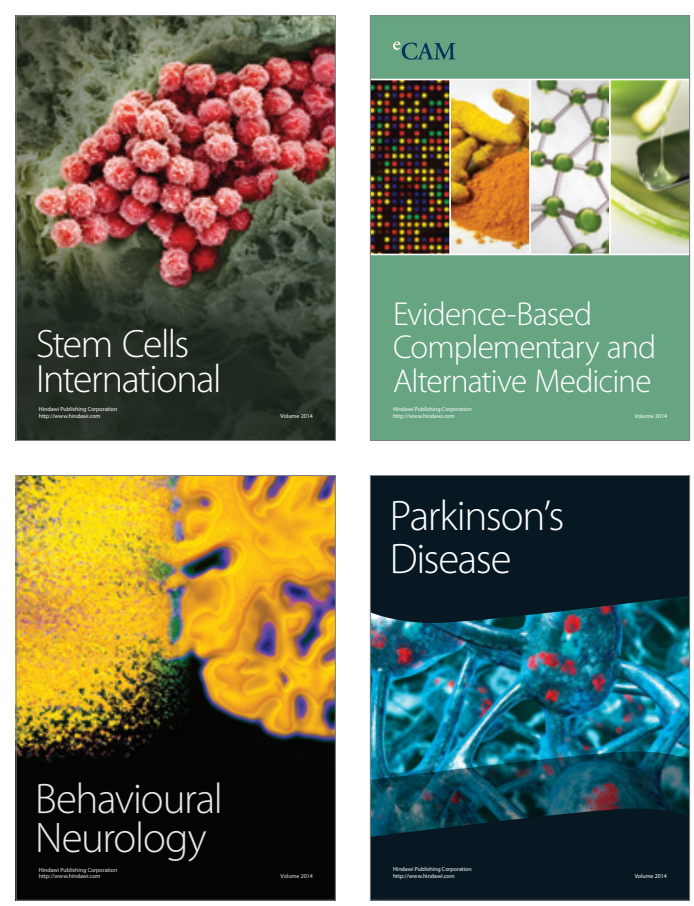

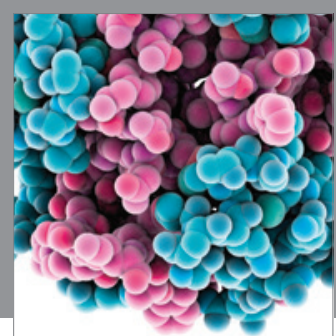

Journal of
Diabetes Research

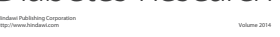

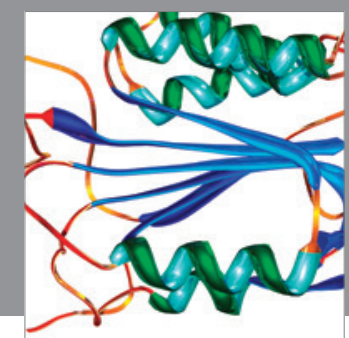

Disease Markers
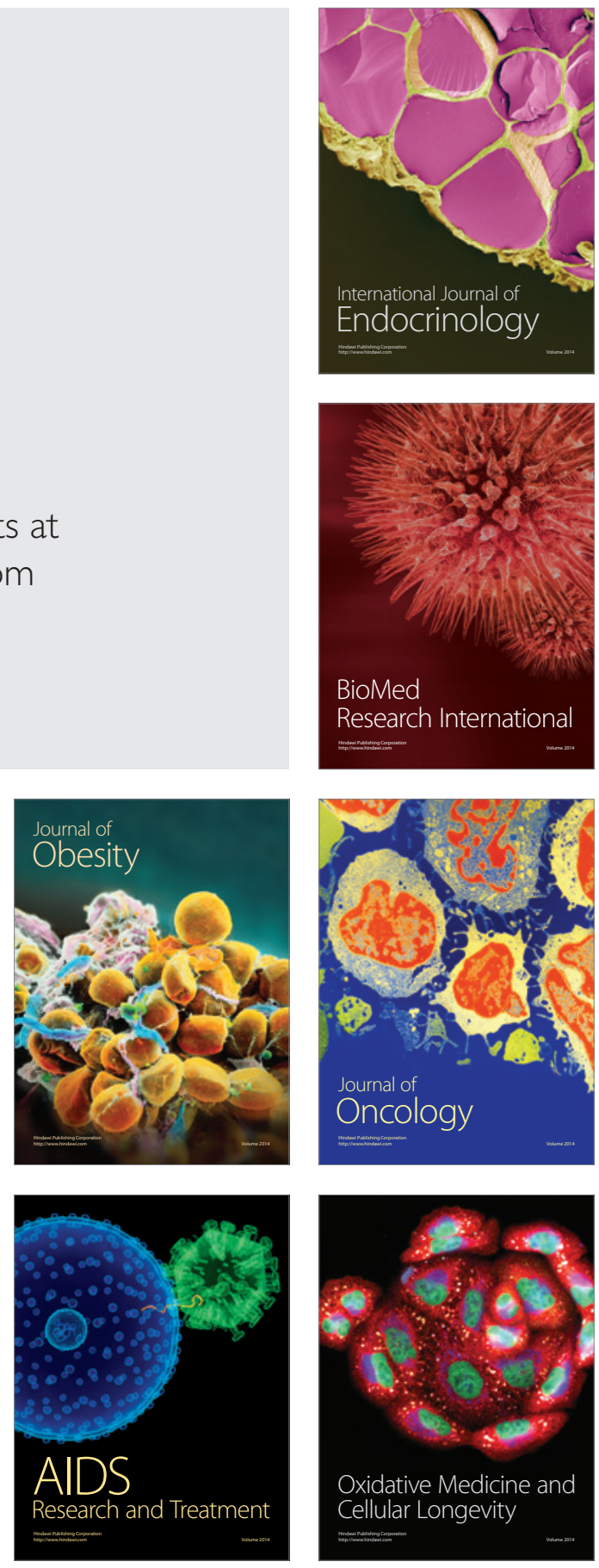